\title{
Effets de trois traitements culinaires sur les propriétés physico-chimiques, fonctionnelles et anti-oxydantes de l'igname sauvage (Dioscorea praehensilis Benth) cultivé dans la région de l'Est-Cameroun
}

\author{
Serge Ricardo POBO KENFACK ${ }^{1 *}$, Mama MOUAMFON ${ }^{2,3}$, Stephano TAMBO TENE ${ }^{1}$, \\ Jules Romain NGUEGUIM ${ }^{4}$ et Youchahou POUTOUGNIGNI MATENCHI ${ }^{5}$
}

\author{
${ }^{1}$ Unité de Recherche de Biochimie des Plantes Médicinales, des Sciences Alimentaires et de Nutrition, \\ Département de Biochimie, Faculté des Sciences, Université de Dschang B.P. 67 Dschang, Cameroun. \\ ${ }^{2}$ Fondation Camerounaise de la Terre Vivante (FCTV), Cameroun. \\ ${ }^{3}$ Landscape Conservation \& Development (LCD), Cameroun. \\ ${ }^{4}$ Institut de Recherche Agricole pour le Développement (IRAD), Cameroun. \\ ${ }^{5}$ Institute of advanced science, Ege University, Turquie. \\ *Auteurs correspondants ; E-mail : sergekenfack@yahoo.com; \\ Tél. : (+237) 6975394 75/(+237) 670793732
}

Received: 31-08-2021

Accepted: 15-12-2021

Published: 31-12-2021

\section{RESUME}

Le «SAPA» (Dioscorea praehensilis Benth) est une espèce dont les liens socio-culturels conservés avec les Baka ne sont plus à démontrer. Son utilisation dans cette zone passe par plusieurs traitements culinaires (ébullition, braise...) qui affecte certaines propriétés jusqu'ici non encore élucidées. Le présent travail était axé sur la valorisation de D. praehensilis au Cameroun par l'étude de l'effet de quelques traitements culinaires sur ses propriétés physico-chimiques, fonctionnelles et anti-oxydantes. Le matériel végétal (échantillons cru, bouilli, braisé et cuit sous cendre), une fois séché et broyé a subi plusieurs analyses physico-chimiques, fonctionnelles et anti-oxydantes suivant des méthodes AOAC connues. Les résultats ont révélé que les teneurs en amidon et en amylose étaient élevées dans les échantillons bouillis $(26,63 \pm 5,11)$ et ceux cuits sous cendre $(55,49 \pm 0,25)$ contrairement à l'amylopectine. La valeur la plus élevée en sucre réducteur a été obtenue par cuisson à la braise $(0,85 \pm 0,15)$ et la plus faible par ébullition $(0,30 \pm 0,00)$. La teneur en saponines variait entre 0,77 (bouilli) et 1,70 $\mathrm{mg} / 100 \mathrm{~g}$ de MS (cru et braise). L'échantillon cru a présenté une teneur en phytates plus élevée (18,30 mg/100 $\mathrm{g}$ de MS) alors que ceux bouillis et cuits sous cendre les valeurs les plus faibles $(6,10 \mathrm{mg} / 100 \mathrm{~g}$ de MS) contrairement aux oxalates où on n'a pas observé de différence significative $(P<0,05)$ entre traitements. Au regard des résultats, il ressort que les propriétés de $D$. praehensilis sont influencées par le traitement culinaire. Ainsi, la valorisation du «SAPA » au Cameroun pourrait prendre en compte ses caractéristiques culinaires. (C) 2021 International Formulae Group. All rights reserved.

Mots clés: Baka, D. praehensilis, igname sauvage, variabilité physicochimique et antinutritionnelle, traitement culinaire, vulgarisation. 


\title{
Effects of three culinary treatments on the physicochemical, functional and antioxidant properties of wild yam (Dioscorea praehensilis Benth) grown in the East Cameroon region
}

\begin{abstract}
The 'SAPA' (Dioscorea praehensilis Benth) is a species whose socio-cultural links with the Baka are well established. Its use in this area involves several culinary treatments (boiling, braising, etc.) which affect certain properties that have not yet been elucidated. The present work focused on the valorization of $D$. praehensilis in Cameroon by studying the effect of some culinary treatments on its physicochemical, functional and antioxidant properties. The plant material (raw, boiled, braised and ash-cooked samples), once dried and ground, underwent several physicochemical, functional and antioxidant analyses according to known AOAC methods. The results revealed that starch and amylose contents were high in the boiled (26.63 \pm 5.11$)$ and ashbaked $(55.49 \pm 0.25)$ samples, while amylopectin was low. The highest reducing sugar value was obtained by braising $(0.85 \pm 0.15)$ and the lowest by boiling $(0.30 \pm 0.00)$. The saponin content varied between 0.77 (boiled) and $1.70 \mathrm{mg} / 100 \mathrm{~g}$ DM (raw and ember). The raw sample showed a higher phytate content (18.30 mg/100 g DM) while the boiled and baked samples showed the lowest values $(6.10 \mathrm{mg} / 100 \mathrm{~g} \mathrm{DM})$, unlike oxalates where no significant difference $(\mathrm{P}<0.05)$ was observed between treatments. The results show that the properties of $D$. praehensilis are influenced by the culinary treatment. Thus, the valorisation of "SAPA" in Cameroon could take into account its culinary characteristics.
\end{abstract}

(C) 2021 International Formulae Group. All rights reserved.

Keywords: Baka, D. praehensilis, wild yam, physicochemical and anti-nutritional variability, culinary treatment, popularization.

\section{INTRODUCTION}

Parmi les plantes racinaires à tubercules, les ignames, appartenant au genre Dioscorea sont cultivées sur toute l'étendue de la planète (zone tropicale, subtropicale et tempérée) et comptent environ 600 espèces repartir sur la surface du globe dont 90 d'entre elles sont estimées comestibles (Shekhar et al., 2015; Doussoh et al., 2016). Avec une production annuelle estimée à 556647 tonnes en 2013, l'igname représente après le manioc et le macabo/taro, la troisième plante racinaire à tubercules produite au Cameroun (INS, 2015). Elle est généralement consommée sous la forme bouillie et transformée également en cossettes. L'identification de l'igname comme espèce prioritaire à la domestication est corrélée à son importance qui reste encore secondaire, bien qu'elle apporte une contribution protéinique substantielle à l'alimentation, se plaçant avant les sources de protéines animales (Sanginga et Mbabu, 2015). Cette contribution optimiserait la valeur et le rôle de cette espèce de seconde importance tout en améliorant sa production en fonction des désirs des paysans et des attentes du marché. Elle favorise ainsi sa conservation en milieu naturel et son insertion dans des systèmes de cultures et améliore sa qualité et quantité en termes de production et d'apport alimentaire.

De nombreuses études portant sur la caractérisation et les tests organoleptiques de certaines ignames ont été faites en vue de leur domestication. Toutefois, des études ont rapproché la valeur nutritionnelle de l'igname à celle de la pomme de terre avec une teneur élevée en matières azotées (Medoua et al., 2007). Parmi les espèces recensées du genre Dioscorea, figure $D$. praehensilis dont la caractérisation et la dénomination ont été effectuées par les Baka à l'Est-Cameroun (Dounias, 1996). D. praehensilis est une espèce se trouvant dans les forêts secondaires et des vieilles jachères dans la région de l'Est Cameroun et dont l'intérêt pour les Baka est très capital. C'est une plante grimpante dont l'axe épineux présente des cataphylles (feuilles réduites) opposées et des rameaux axillaires opposés. Le tubercule de forme cylindrique, très allongé verticalement avec une chair 
violacée ou blanche recouverte d'une peau légèrement fine et des vieilles racines épineuses à sa tête se trouve à au moins 1,50 $\mathrm{m}$ de profondeur.

Les Baka de l'Est Cameroun sont un peuple dont l'une des cultures ancestrales est le SAPA, bien que ce dernier tend à disparaitre dans leur habitat à cause de nombreuses pressions anthropiques sur les populations de son espèce. Il est généralement consommé par ce peuple sous forme bouillie, braisée ou cuit à la cendre. De ce fait, les liens socio-culturels conservés entre les Baka et le SAPA qui, constituait jadis leur base alimentaire contrairement aux autres tubercules justifieraient l'intérêt et la nécessité de développer des recherches concernant le $D$. praehensilis. D'où l'objectif de cette étude qui était de contribuer à la valorisation du «SAPA» au Cameroun à travers la caractérisation de ses propriétés physicochimiques et antinutritionnelles en fonction des différents traitements culinaires.

\section{MATERIEL ET METHODES}

\section{Présentation de la zone d'étude}

L'étude a été réalisée dans la communauté BAKA du village Payo, située à environ $15 \mathrm{~km}$ de la ville de Lomié, Arrondissement de Lomié, Département du Haut-Nyong, Région de l'Est Cameroun. Le village Payo se trouve dans une zone de forêts humides à pluviométrie bimodale avec quatre (04) saisons. Le climat chaud et humide qu'offre cette zone est de type "équatorial guinéen", avec des températures annuelles qui se trouvent autour de $24^{\circ} \mathrm{C}$ en moyenne et une pluviométrie de $1300-1800 \mathrm{~mm}$ par an, repartie en deux saisons humides bien distinctes (Philippart et Feteke, 2008). Le choix de cette localité se justifie par la forte présence de la population Baka dans un village Baka autonome avec une habitude alimentaire orientée vers la collecte et la consommation de cette igname. De même, au cours de la sélection des espèces potentiellement domesticable dans le village Payo, le SAPA a été identifié comme espèce prioritaire à la domestication par les membres de la communauté.

\section{Matériel Biologique}

Dioscorea praehensilis Benth ou SAPA en langue Baka, de la Famille des Dioscoreaceae est le matériel végétal utilisé dans le cadre de ce travail a été récolté dans la région de l'Est Cameroun, Arrondissement de Lomié, Village Payo et ses environs. Il a été principalement récolté en forêts secondaires jeunes et les vieilles jachères. La récolte $d u$ germoplasme du $D$. praehensilis a été faite au mois de décembre par déterrage.

\section{Méthodologie \\ Préparation des farines}

Les ignames obtenues comme citées précédemment ont été convenablement nettoyées à l'eau de robinet puis divisée en quatre lots. Le premier lot constituait l'échantillon cru qui a été pelé, le deuxième lot a subi la cuisson par ébullition avant d'être pelé, le troisième quant à lui a été braisé et pelé et en fin le quatrième a subi une la cuisson à la cendre. Après, ils ont été découpés en dés fins à l'aide d'un couteau de cuisine puis sécher à l'étuve à $45^{\circ} \mathrm{C}$ pendant $72 \mathrm{~h}$. Après séchage, les cossettes obtenues ont été broyées à l'aide d'un robot-mixeur (Polymixa) jusqu'à obtention des poudres, lesquelles ont été mise dans des sachets en polyéthylène puis conservées dans un dessiccateur.

\section{Analyses}

La détermination de la teneur en eau a été faite suivant la méthode décrite par AFNOR (1981). Dans un extracteur de Soxhlet, les lipides de l'échantillon ont été entrainés dans un solvant organique puis après séchage, la masse d'huile a été déduite selon le protocole de l'AFNOR (1981). La teneur en phénols totaux des différents échantillons a été déterminée par la méthode spectrophotométrique utilisant le réactif de Folin-Ciocalteu comme décrite par Gao et al., (2000). La détermination de la teneur en saponines a été faite par la méthode de Koziol (1991). Le pH et l'acidité titrable ont été évalué selon le protocole décrit par l'AFNOR (1981). En effet, $10 \mathrm{~g}$ de farine sèche de chaque échantillon ont été mélangés à $100 \mathrm{ml}$ d'eau distillée puis le $\mathrm{pH}$ a été directement lu sur la suspension puis titré avec du NaOH 0,1 N par la suite. La teneur en flavonoïdes a été 
déterminée selon la méthode colorimétrique décrite par Bahorun et al. (2006). En ce qui concerne la teneur en phytates, elle a été déterminée par la méthode titimétrique au $\mathrm{Fecl}_{3}, 6 \mathrm{H}_{2} \mathrm{O}$ de Reddy et al. (1989). L'activité émulsifiante quant à elle a été déterminée suivant la méthode de Beuchat et al. (1977), tandis que la stabilité des émulsions a été évaluée selon Pearce et Kinsella (1978). La densité massique a été déterminée suivant la méthode décrite par Okaka et al. (1991) et pour la détermination de la teneur en amidon, le dosage a été réalisé selon la méthode décrite par Jarvis et Walker (1993). La température de gélatinisation a été déterminée à partir la méthode de Cabrera et al. (1984). La Capacité de Rétention d'Eau (CRE) par la méthode décrite par Leach et Schoch (1959). Les tanins hydrolysables réagissent avec le chlorure ferrique et donnent une coloration bleue mesurée par spectrophotométrie. Le dosage de l'acide cyanhydrique a été effectué selon la méthode alcaline de titration. La concentration en Frap a été faite par la méthode décrite par Oyaizu (1986). L'activité antioxydante des différents extraits a été évaluée par la méthode utilisant le DPPH (2,2-diphényl-1-picryl1hydrazyl). La concentration en $\mathrm{RoH}$ a été déterminée telle que décrite par Marfak (2003). Le gonflement a été effectué par la méthode décrite par Okezie et Bello (1988).

\section{Traitement et analyses statistiques des données}

Les résultats de la composition chimique obtenus ont été exprimés en moyenne \pm écart type calculés et présentés sous forme de tableau avec le logiciel SPSS version 20, analysés par le test de variance (ANOVA) au seuil de probabilité 5\%. Par la suite, une analyse en composante principale (ACP) a été réalisée à l'aide du logiciel XLSTAT version 2007, extension du logiciel EXCEL 13 pour le regroupement de la composition des ignames en se basant sur la composition chimique par traitement culinaires. L'Analyse en Composantes Principales (ACP) a permis de voir le nombre adéquat de classes dans lesquelles les données sont progressivement regroupées, ainsi que leurs compositions en fonction du traitement culinaire des ignames.

\section{RESULTATS}

Effets des différents traitements culinaires sur la composition chimique approximative du "Sapa"

Le Tableau 1 présente la composition chimique approximative des ignames sauvages ayant subi différents traitements culinaires. Il ressort de ce tableau que la teneur en eau variait de $2,07 \%$ (braisé) à $5,32 \%$ (bouilli). Elle a été significativement $(p<0,05)$ abaissée par les cuissons à la braise et à la cendre mais par contre augmenté par la cuisson par ébullition. De même, il ressort également de ce tableau que la matière sèche des échantillons variait dans le sens inverse de la teneur en eau ( $r=-$ $1,000 ; \mathrm{p}<0,01)$.

Les lipides jouent un rôle très important dans la régulation de la température corporelle, la constitution des membranes cellulaires et la protection de l'organisme. Sa valeur était comprise entre $4,67 \%$ et $6,04 \%$. Elle n'a pas été significativement affectée par les traitements ( $p>0,05)$. La valeur la plus faible a été obtenue avec l'échantillon cuit par ébullition.

La teneur du cyanure qui est un antinutriment synthétisé par les racines et tubercules pour se protéger contre les facteurs environnementaux était comprise entre 5,88$8,91 \mathrm{mg}$ de $\mathrm{HCN} / \mathrm{kg}$ de MS (Tableau 1). Cette valeur a été significativement abaissée par la braise $(\mathrm{p}<0,05)$.

La teneur en amidon et en amylose ont été significativement augmentés par l'ébullition et la cuisson à la cendre contrairement à l'amylopectine. Pour ce qui est de la teneur en sucres réducteurs, la valeur la plus élevée a été obtenue avec l'échantillon cuit à la braise et la plus faible avec celle cuit par ébullition (Tableau 1).

Effets des différents traitements culinaires sur la composition approximative en antinutriments et composés biologiquement actifs du "Sapa"

La composition approximative en quelques antinutriments et composes biologiquement actifs de l'igname sauvage a été étudiée. La teneur en saponines variait entre 0,77 (cuisson par ébullition) et $1,70 \mathrm{mg} / 100 \mathrm{~g}$ de MS (cru et braise). Ce paramètre a été significativement réduit par l'ébullition 
(Tableau 2). Néanmoins, ce phénomène n'a pas été observé lors de la braise et de la cuisson à la cendre. Les teneurs en composés phénoliques et en flavonoïdes exprimées en $\mathrm{mg}$ EAG/100 g de MS et en mg ECAT/100 g de MS respectivement sont également présenté (Tableau 2). Il ressort du Tableau 2 que l'échantillon cru a présenté la teneur en phytates la plus élevée $(18,30 \mathrm{mg} / 100 \mathrm{~g}$ de MS) alors que ceux bouillis et cuits à la cendre les valeurs les plus faibles $(6,10 \mathrm{mg} / 100 \mathrm{~g}$ de MS$)$. Pour ce qui est des oxalates, il n'a pas été observé de différence significative entre les traitements mais plutôt avec l'échantillon cru. La teneur en tannin variait de 0,00 à 0,37 $\mathrm{mg} / 100 \mathrm{~g}$ de MS. Elle est significativement $(p<0.05)$ basse dans les échantillons cuits par par ébullition et par la cendre.

Effet des traitements culinaires sur la capacité inhibitrice du radical hydroxyle

La capacité inhibitrice du radical hydroxyle des extraits des différents échantillons croit avec la concentration en extrait jusqu'à atteindre son maximum à 200 $\mu \mathrm{g} / \mathrm{ml}$ (Figure 1). L'extrait issu de l'échantillon cru a présenté la plus faible capacité inhibitrice contrairement à l'échantillon braisé qui a présenté le plus fort pouvoir inhibiteur. En outre les différents traitements culinaires ont amélioré la capacité inhibitrice du radical hydroxyl.

Effet des traitements culinaires sur le pouvoir réducteur de l'ion fer III $\left(\mathrm{Fe}^{3+}\right)$

Le pouvoir réducteur des extraits des échantillons crus, bouillis et braisés croit avec la concentration jusqu'à atteindre le pic à la concentration la plus élevée (Figure 2). Pour ce qui est de l'échantillon braisé, le pouvoir réducteur du $\mathrm{Fe}^{3+}$ optimal a été observé à une concentration de $50 \mu \mathrm{g} / \mathrm{ml}$.

\section{Indicateur non spécifique de la réaction de Maillard développé lors des traitements culinaires}

$\mathrm{Au}$ vu des réactions de brunissement non enzymatique effectuées selon les traitements culinaires sur les ignames, l'absorbance de l'échantillon cru à $420 \mathrm{~nm}$ était la plus importante suivit de l'échantillon cuit à la cendre (Figure 3); ce qui indique une abondance des mélanoïdines dans ces échantillons. Ce paramètre a été significativement $(\mathrm{p}<0.05)$ abaissé par le traitement avec la valeur la plus faible observée dans l'échantillon bouilli.

\section{Caractérisation physique et fonctionnelle des différents échantillons \\ Caractérisation physique}

Les résultats obtenus montrent que le pH était situé entre 5,30 pour l'échantillon cru et 5,64 pour l'échantillon cuit à la cendre. Ce paramètre n'a pas été significativement $(p>0,05)$ affecté par le traitement culinaire (Tableau 3). Le $\mathrm{pH}$ des farines a une influence sur la capacité de rétention d'eau et leur vitesse de rétrogradation. Il est également un paramètre important dans la production des gels d'amidon de viscosité élevée. Les résultats de l'acidité titrable montrent que ce paramètre a été significativement $(\mathrm{p}<0,05)$ élevé par les traitements culinaires mais sans variation entre les traitements. La densité massique représente le rapport entre la masse et le volume occupé par un aliment et influence sur le conditionnement et la prise alimentaire. Ce paramètre variait entre $0,93 \mathrm{~g} / \mathrm{ml}$ pour l'échantillon cru à $1,09 \mathrm{~g} / \mathrm{ml}$ pour l'échantillon braisé (Tableau 4). Il a été significativement $(\mathrm{p}<0,05)$ augmenté par la braise et la cuisson à la cendre.

\section{Capacité de Rétention d'eau (CRE)}

La quantité d'eau retenue au cours de la gélatinisation augmentait avec la température pour les différents échantillons (Figure 4). Entre $70-80^{\circ} \mathrm{C}$, il a été observé une brusque augmentation de la capacité de rétention d'eau dans tous les échantillons jusqu'à atteindre le maximum à $100^{\circ} \mathrm{C}$. Cette température correspondrait à la température au-delà de laquelle on assisterait à une chute de la rétention d'eau marquée par une rupture des interactions eau-amidon.

\section{Taux de gonflement $(\mathrm{Tg})$}

Le taux de gonflement croit proportionnellement avec la température jusqu'à un maximum de $100{ }^{\circ} \mathrm{C}$ pour les échantillons cru, braisé et cuit à la cendre (Figure 5). 


\section{Capacité de Rétention d'Huile (CRH)}

La capacité de rétention d'huile met en évidence les interactions hydrophobes pouvant être établies par les constituants de la farine. Cette propriété est influencée par la composition nutritionnelle (lipides, protéines, sucres réducteurs et phénols) et la taille des particules. La capacité de rétention d'huile du SAPA croit jusqu'à $40^{\circ} \mathrm{C}$ pour tous les échantillons et décroit à $50^{\circ} \mathrm{C}$ avant de recroitre jusqu'à $100^{\circ} \mathrm{C}$ (Figure 6).

\section{Influence des traitements culinaires sur les propriétés fonctionnelles et anti-oxydantes : classification hiérarchique ascendante (CAH)}

La classification hiérarchique ascendante des variables présentée par la Figure $7 \mathrm{a}$ permet de visualiser 3 groupes montrant que les variables sont corrélés par petit groupe. Il ressort en outre de la Figure 7b portant sur la classification hiérarchique des observations que celles-ci sont subdivisées en 3 classes. La première classe était formée de l'échantillon bouilli, la seconde de l'échantillon cru, la troisième des ignames braisées et cuites à la cendre. Ces résultats confirment les caractéristiques particulières de chaque traitement. En effet, ce regroupement montre que la cuisson à la cendre et à la braise possèdent les mêmes propriétés. Outre cela, il ressort que les traitements ont significativement affectés les propriétés des différentes ignames. Tambo et al. (2019a,b) ont également observé une variation significative des propriétés physico-chimiques et fonctionnelles dans la farine de manioc à la suite de la torréfaction. Il ressort du graphe de classification des variables que le premier groupe était formé par la Capacité Réductrice du Fer (FRAP), la CRE, la TE, les phytates, le taux de gonflement, l'amylopectine, la réaction de maillard, les oxalates, le cyanure et l'indice de reconstitution. Le deuxième regroupement était constitué de la matière sèche, sucres réducteurs $(\mathrm{SR})$, tanins, saponines et de la stabilité des émulsions. La stabilité des émulsions est positivement corrélée aux tanins $(r=0,468)$, saponines $(r=0,697)$ et $\mathrm{SR}(\mathrm{r}=$ $0,969)$. Le troisième groupe était formé par l'amidon, l'amylose, la Capacité Inhibitrice du Radical Hydroxyl (ROH), les lipides, les flavonoïdes, la température de gélatinisation, l'émulsion, le $\mathrm{pH}$, les phénols, la densité massique, l'acidité titrable et la CRH. La capacité inhibitrice du radical hydroxyle est positivement corrélée à la teneur en lipides $(\mathrm{r}=$ $0,690)$, au $\mathrm{pH}(\mathrm{r}=0,946)$, aux phénols totaux $(\mathrm{r}=0,544)$ et aux flavonoïdes $(\mathrm{r}=0,478)$.

Tableau 1: Effets des différents traitements culinaires sur la composition chimique approximative de l'igname sauvage.

\begin{tabular}{lllll}
\hline Flour & Cru & Bouilli & Braisé & Cendre \\
\hline Teneur en eau (\%) & $3,80 \pm 0,34^{\mathrm{b}}$ & $5,32 \pm 0,26^{\mathrm{c}}$ & $2,07 \pm 0,41^{\mathrm{a}}$ & $2,48 \pm 0,02^{\mathrm{a}}$ \\
\hline Matière sèche (MS) (\%) & $96,19 \pm 0,34^{\mathrm{b}}$ & $94,67 \pm 0.26^{\mathrm{a}}$ & $97,93 \pm 0,41^{\mathrm{c}}$ & $97,52 \pm 0,028^{\mathrm{c}}$ \\
\hline Lipides (\%) & $5,00 \pm 0,09^{\mathrm{ab}}$ & $4,60 \pm 0,39^{\mathrm{a}}$ & $5,39 \pm 0,30^{\mathrm{ab}}$ & $6,04 \pm 0,52^{\mathrm{b}}$ \\
\hline Cyanure (mg de HCN/kg de MS) & $8,91 \pm 1,14^{\mathrm{b}}$ & $7,07 \pm 0,38^{\mathrm{ab}}$ & $5,88 \pm 0,53^{\mathrm{a}}$ & $7,45 \pm 0,15^{\mathrm{ab}}$ \\
\hline Amidon (\%) & $17,48 \pm 2,01^{\mathrm{a}}$ & $26,63 \pm 5,11^{\mathrm{b}}$ & $10,50 \pm 0,04^{\mathrm{a}}$ & $55,49 \pm 0,25^{\mathrm{c}}$ \\
\hline Amylose (\% d'amidon) & $4,62 \pm 0,22^{\mathrm{a}}$ & $9,71 \pm 3,13^{\mathrm{b}}$ & $3,52 \pm 0,73^{\mathrm{a}}$ & $40,76 \pm 0,73^{\mathrm{c}}$ \\
\hline Amylopectine (\% d'amidon) & $95,37 \pm 0,22^{\mathrm{c}}$ & $90,28 \pm 3,13^{\mathrm{b}}$ & $96,47 \pm 0,73^{\mathrm{c}}$ & $59,23 \pm 0,73^{\mathrm{a}}$ \\
\hline Sucres réducteurs (\%) & $0,46 \pm 0,13^{\mathrm{ab}}$ & $0,30 \pm 0,00^{\mathrm{a}}$ & $0,85 \pm 0,15^{\mathrm{b}}$ & $0,47 \pm 0,09^{\mathrm{ab}}$ \\
\hline $\begin{array}{l}\text { Les valeurs présentées sous forme de moyennes }(\mathrm{n}=3) \pm \text { écart type portant les mêmes lettres en exposant }(\mathrm{a}, \mathrm{b}, \mathrm{c}, \ldots) \text { dans la } \\
\text { même ligne ne sont pas significativement différents au seuil de 5\%. }\end{array}$ & & &
\end{tabular}


Tableau 2 : Effets des différents traitements culinaires sur la composition approximative en quelques antinutriments et composes biologiquements actifs de l'igname sauvage.

\begin{tabular}{lcccc}
\hline Flour & Cru & Bouillie & Braisé & Cendre \\
\hline Saponin (mg/100 g de MS) & $1,70 \pm 0,06^{\mathrm{b}}$ & $0,77 \pm 0,03^{\mathrm{a}}$ & $1,70 \pm 0,18^{\mathrm{b}}$ & $1,48 \pm 0,1^{\mathrm{b}}$ \\
\hline Phénol (mg EAG/100 g de MS) & $1,14 \pm 0,13^{\mathrm{a}}$ & $3,60 \pm 0,05^{\mathrm{b}}$ & $3,27 \pm 0,26^{\mathrm{b}}$ & $3,91 \pm 0,42^{\mathrm{b}}$ \\
\hline Phytates (mg/100 g de MS) & $18,30 \pm 0,00^{\mathrm{c}}$ & $6,10 \pm 0,00^{\mathrm{a}}$ & $10,67 \pm 2,15^{\mathrm{b}}$ & $6,10 \pm 0,00^{\mathrm{a}}$ \\
\hline Oxalates (mg/100 g de MS) & $0,56 \pm 0,15^{\mathrm{b}}$ & $0,22 \pm 0,00^{\mathrm{a}}$ & $0,22 \pm 0,00^{\mathrm{a}}$ & $0,22 \pm 0,00^{\mathrm{a}}$ \\
\hline Flavonoïdes (mg ECAT/100 g de MS) & $0,69 \pm 0,07^{\mathrm{a}}$ & $2,68 \pm 0,35^{\mathrm{c}}$ & $1,96 \pm 0,08^{\mathrm{b}}$ & $1,99 \pm 0,20^{\mathrm{b}}$ \\
\hline Tanins (mg ECAT/100 g de MS) & $0,37 \pm 0,02^{\mathrm{b}}$ & $0,00 \pm 0,00^{\mathrm{a}}$ & $0,34 \pm 0,02^{\mathrm{b}}$ & $0,00 \pm 0,00^{\mathrm{a}}$
\end{tabular}

Les valeurs présentées sous forme de moyennes $(\mathrm{n}=3) \pm$ écart type portant les mêmes lettres en exposant $(\mathrm{a}, \mathrm{b}, \mathrm{c}$,$) dans la$ même ligne ne sont pas significativement différents au seuil de $5 \%$.

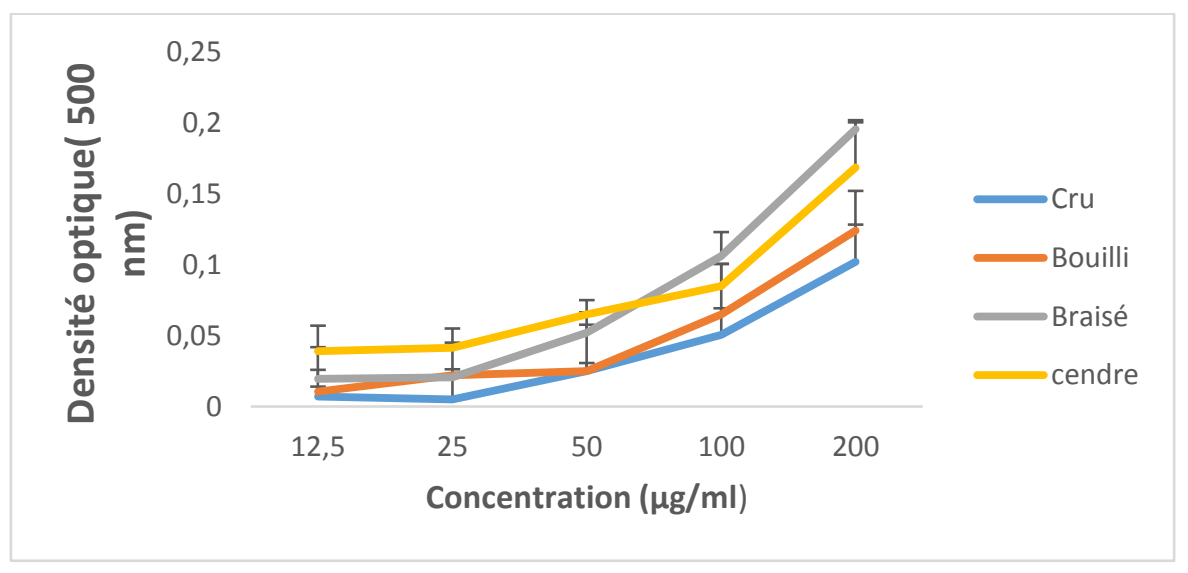

Figure 1 : Effet des traitements culinaires sur la capacité inhibitrice du radical hydroxyle à différentes concentrations.

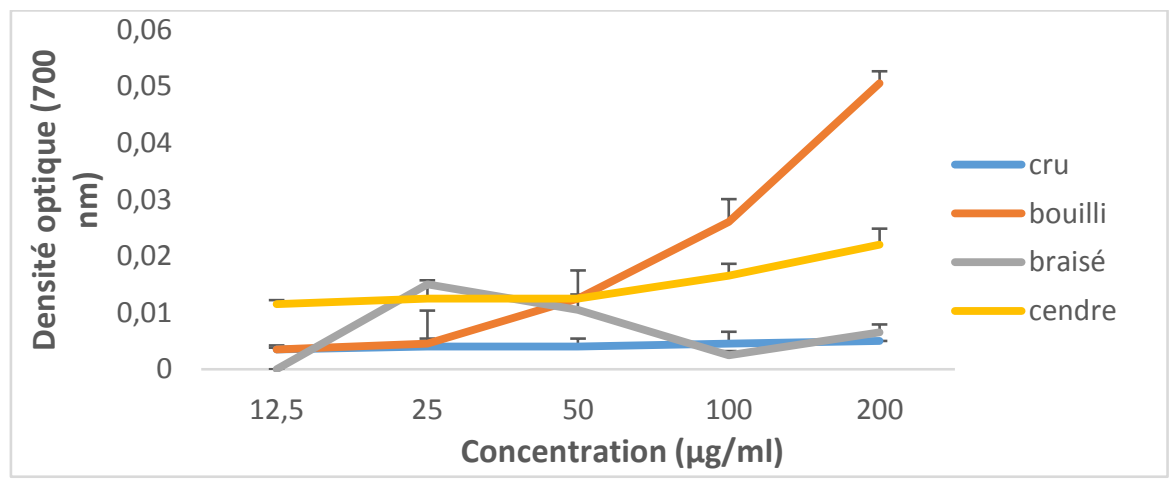

Figure 2: Effet des traitements culinaires sur le pouvoir réducteur de l'ion fer III à différentes concentrations. 


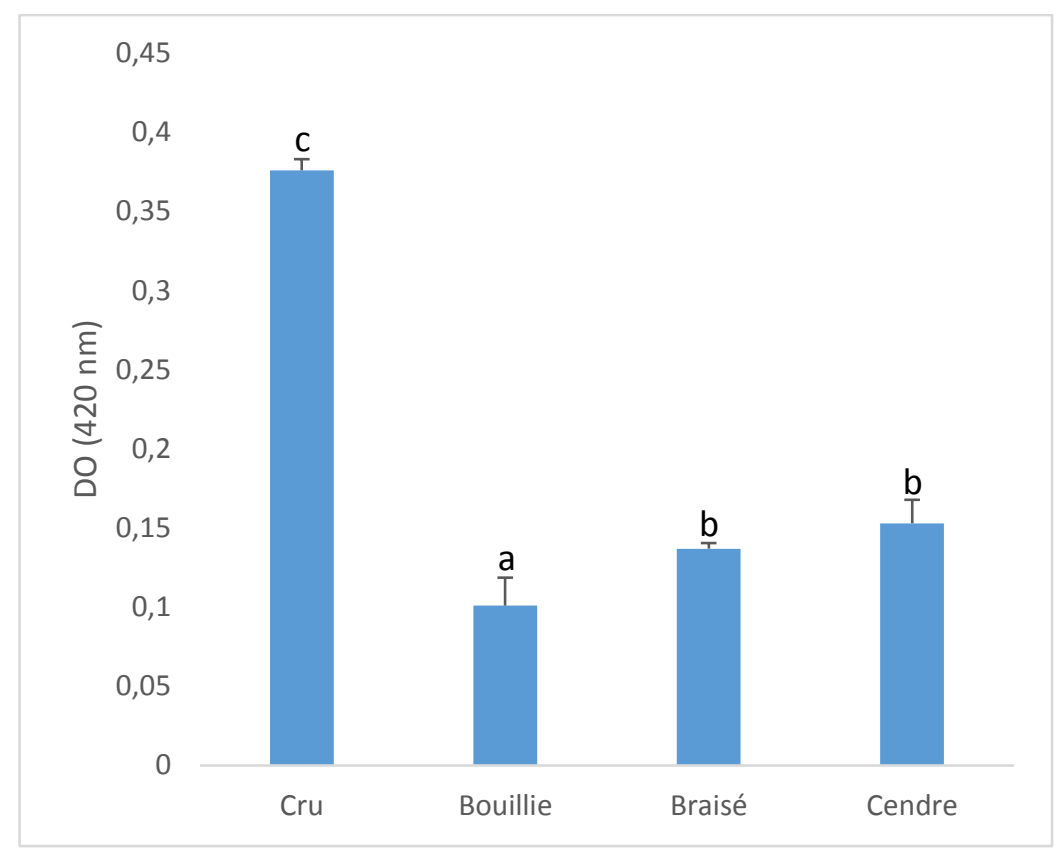

Figure 3 : Effet des traitements culinaires sur l'absorbance à $420 \mathrm{~nm}$ comme indice non spécifique de la reaction de Maillard.

Les valeurs présentées sous forme de moyennes $(n=3) \pm$ écart type portant les mêmes lettres en exposant $(a, b, c)$ ne sont pas significativement différents au seuil de $5 \%$.

Tableau 3 : Effets des différents traitements culinaires sur les propriétés physiques de l'igname sauvage.

\begin{tabular}{lllll}
\hline Flour & Cru & Bouillie & Braisé & Cendre \\
\hline PH & $5,30 \pm 0,00^{\mathrm{a}}$ & $5,37 \pm 0,48^{\mathrm{a}}$ & $5,62 \pm 0,01^{\mathrm{a}}$ & $5,64 \pm 0,00^{\mathrm{a}}$ \\
\hline Acidité titrable & $3,25 \pm 1,34^{\mathrm{a}}$ & $7,10 \pm 0,56^{\mathrm{b}}$ & $7,40 \pm 1,41^{\mathrm{b}}$ & $7,50 \pm 0,42^{\mathrm{b}}$ \\
\hline Densité massique (g/ml) & $0,93 \pm 0,02^{\mathrm{a}}$ & $0,99 \pm 0,05^{\mathrm{ab}}$ & $1,09 \pm 0,02^{\mathrm{c}}$ & $1,06 \pm 0,00 \mathrm{~b}^{\mathrm{c}}$ \\
\hline Emulsion (\%) & $56,66 \pm 4,71^{\mathrm{a}}$ & $66,66 \pm 0,00^{\mathrm{b}}$ & $76,66 \pm 0,00^{\mathrm{c}}$ & $73,33 \pm 0,00^{\mathrm{c}}$ \\
\hline Stabilité des émulsions $(\boldsymbol{\%})$ & $68,33 \pm 2,35^{\mathrm{a}}$ & $65,00 \pm 0,00^{\mathrm{a}}$ & $78,33 \pm 2,35^{\mathrm{b}}$ & $71,66 \pm 2,35^{\mathrm{a}}$ \\
\hline Température de gélatinisation $\left({ }^{\circ} \mathbf{C}\right)$ & $72,00 \pm 0,70^{\mathrm{a}}$ & $72,35 \pm 0,91^{\mathrm{a}}$ & $72,75 \pm 3,18^{\mathrm{a}}$ & $76,00 \pm 1,41^{\mathrm{a}}$ \\
\hline Indice de reconstitution $(\mathbf{I R})(\boldsymbol{\%})$ & $73,65 \pm 3,98^{\mathrm{b}}$ & $64,13 \pm 9,11^{\mathrm{ab}}$ & $52,27 \pm 3,21^{\mathrm{a}}$ & $53,47 \pm 0,74^{\mathrm{a}}$ \\
\hline
\end{tabular}

Les valeurs présentées sous forme de moyennes $(n=3) \pm$ écart type portant les mêmes lettres en exposant $(a, b, c, \ldots)$ dans la même ligne ne sont pas significativement différents au seuil de $5 \%$. 


\section{S. R. POBO KENFACK et al. / Int. J. Biol. Chem. Sci. 15(6): 2665-2684, 2021}

Tableau 4 : Matrix de corrélation de pearson (r) entre les propriétés physico-chimiques, fonctionnelles et anti-oxydantes.

\begin{tabular}{|c|c|c|c|c|c|c|c|c|c|c|c|c|c|c|c|c|c|c|c|c|c|c|c|c|c|c|c|}
\hline$\frac{\frac{n}{0}}{\frac{\pi}{\pi}}$ & 될 & $\sum^{\infty}$ & 光 & 趈 & 竞 & E & $\begin{array}{l}\bar{\pi} \\
\text { 己े }\end{array}$ & 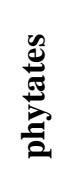 & 主 & 馬 & 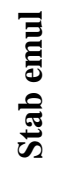 & $\underline{\sim}$ & $\sum$ & $\sum$ & $\stackrel{\theta}{\gtrless}$ & 当 & نَّ & 氞 & 总 & $\stackrel{\mathscr{E}}{2}$ & $\frac{\stackrel{\Xi}{\pi}}{\widetilde{\pi}}$ & 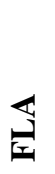 & $\frac{a}{0}$ & 晃 & שِ & $\underset{\mathbb{1}}{\mathbb{\alpha}}$ & $\stackrel{\pi}{0}$ \\
\hline $\mathrm{TE}$ & 1 & & & & & & & & & & & & & & & & & & & & & & & & & & \\
\hline MS & $\begin{array}{l}- \\
\mathbf{1 , 0} \\
\mathbf{0 0 b}\end{array}$ & 1 & & & & & & & & & & & & & & & & & & & & & & & & & \\
\hline LIP & $\begin{array}{l}- \\
0,8 \\
37 b\end{array}$ & 0,837b & 1 & & & & & & & & & & & & & & & & & & & & & & & & \\
\hline SAP & $\begin{array}{l}- \\
\mathbf{0 , 8 0} \\
7 \mathrm{~b}\end{array}$ & $0,807 b$ & $\begin{array}{l}0,5 \\
45 a\end{array}$ & 1 & & & & & & & & & & & & & & & & & & & & & & & \\
\hline $\mathrm{pH}$ & $\begin{array}{l}- \\
0,8 \\
15 b\end{array}$ & $\mathbf{0 , 8 1 5 b}$ & $\begin{array}{l}0,8 \\
33 b\end{array}$ & $\begin{array}{l}0,3 \\
17\end{array}$ & 1 & & & & & & & & & & & & & & & & & & & & & & \\
\hline AT & $\begin{array}{l}- \\
0,2 \\
50\end{array}$ & 0,250 & $\begin{array}{l}0,3 \\
55\end{array}$ & $\begin{array}{l}- \\
0,3 \\
65\end{array}$ & $\begin{array}{l}0,7 \\
51 a\end{array}$ & 1 & & & & & & & & & & & & & & & & & & & & & \\
\hline Cyan & $\begin{array}{l}0,3 \\
56\end{array}$ & $-0,356$ & $\begin{array}{l}- \\
0,1 \\
44 \\
\end{array}$ & $\begin{array}{l}0,1 \\
29\end{array}$ & $\begin{array}{l}- \\
0,6 \\
66 a\end{array}$ & $\begin{array}{l}- \\
0,8 \\
44 b \\
\end{array}$ & 1 & & & & & & & & & & & & & & & & & & & & \\
\hline $\begin{array}{l}\text { phytat } \\
\text { es }\end{array}$ & $\begin{array}{l}- \\
0,0 \\
58 \\
\end{array}$ & 0,058 & $\begin{array}{l}- \\
0,2 \\
39 \\
\end{array}$ & $\begin{array}{l}0,6 \\
31 a\end{array}$ & $\begin{array}{l}- \\
0,5 \\
29 a\end{array}$ & $\begin{array}{l}- \\
0,9 \\
15 b\end{array}$ & $\begin{array}{l}0,5 \\
88 a\end{array}$ & 1 & & & & & & & & & & & & & & & & & & & \\
\hline $\mathrm{DM}$ & $\begin{array}{l}- \\
0,7 \\
16 a\end{array}$ & $\begin{array}{l}0,716 a \\
a\end{array}$ & $\begin{array}{l}0,6 \\
12 a\end{array}$ & $\begin{array}{l}0,1 \\
95\end{array}$ & $\begin{array}{l}0,9 \\
45 b\end{array}$ & $\begin{array}{l}0,8 \\
34 b\end{array}$ & $\begin{array}{l}- \\
\mathbf{0 , 8} \\
\mathbf{7 0 b}\end{array}$ & $\begin{array}{l}- \\
0,5 \\
61 a\end{array}$ & 1 & & & & & & & & & & & & & & & & & & \\
\hline
\end{tabular}


S. R. POBO KENFACK et al. / Int. J. Biol. Chem. Sci. 15(6): 2665-2684, 2021

\begin{tabular}{|c|c|c|c|c|c|c|c|c|c|c|c|c|c|c|c|c|c|c|c|c|c|c|c|c|c|c|c|}
\hline Phe & $\begin{array}{l}0,0 \\
77\end{array}$ & $-0,077$ & $\begin{array}{l}- \\
0,1 \\
92 \\
\end{array}$ & $\begin{array}{l}- \\
0,5 \\
31 a \\
\end{array}$ & $\begin{array}{l}0,3 \\
67\end{array}$ & $\begin{array}{l}0,8 \\
21 b\end{array}$ & $\begin{array}{l}- \\
0,9 \\
02 b\end{array}$ & $\begin{array}{l}- \\
\mathbf{0 , 7} \\
\mathbf{0 5 a}\end{array}$ & $\begin{array}{l}0,6 \\
16 a\end{array}$ & $\begin{array}{l}0,6 \\
96 a\end{array}$ & $\begin{array}{l}0,2 \\
25\end{array}$ & $\begin{array}{l}0,2 \\
29\end{array}$ & $\begin{array}{l}- \\
0,1 \\
57 \\
\end{array}$ & $\begin{array}{l}- \\
0,1 \\
44 \\
\end{array}$ & $\begin{array}{l}0,1 \\
44\end{array}$ & $\begin{array}{l}- \\
0,5 \\
97 a\end{array}$ & $\begin{array}{l}- \\
0,0 \\
65 \\
\end{array}$ & $\begin{array}{l}- \\
0,3 \\
46 \\
\end{array}$ & $\begin{array}{l}- \\
0,9 \\
12 b\end{array}$ & 1 & & & & & & & \\
\hline $\begin{array}{l}\text { oxalat } \\
\text { e }\end{array}$ & $\begin{array}{l}0,1 \\
75\end{array}$ & $-0,175$ & $\begin{array}{l}- \\
0,2 \\
79 \\
\end{array}$ & $\begin{array}{l}0,4 \\
33\end{array}$ & $\begin{array}{l}- \\
0,6 \\
95 a\end{array}$ & $\begin{array}{l}- \\
0,9 \\
97 b\end{array}$ & $\begin{array}{l}0,8 \\
45 b\end{array}$ & $\begin{array}{l}0,9 \\
27 b\end{array}$ & $\begin{array}{l}- \\
0,7 \\
\mathbf{9 6 a} \\
\end{array}$ & $\begin{array}{l}- \\
0,8 \\
82 b\end{array}$ & $\begin{array}{l}- \\
0,2 \\
93 \\
\end{array}$ & $\begin{array}{l}- \\
0,1 \\
69 \\
\end{array}$ & $\begin{array}{l}- \\
0,3 \\
39 \\
\end{array}$ & $\begin{array}{l}- \\
0,3 \\
80 \\
\end{array}$ & $\begin{array}{l}0,3 \\
80\end{array}$ & $\begin{array}{l}0,8 \\
47 \mathrm{~b}\end{array}$ & $\begin{array}{l}- \\
0,4 \\
61\end{array}$ & $\begin{array}{l}0,6 \\
21 a\end{array}$ & $\begin{array}{l}0,9 \\
85 b\end{array}$ & $\begin{array}{l}- \\
0,8 \\
55 b\end{array}$ & 1 & & & & & & \\
\hline FLA & $\begin{array}{l}0,1 \\
65\end{array}$ & $-0,165$ & $\begin{array}{l}- \\
0,1 \\
60\end{array}$ & $\begin{array}{l}- \\
0,6 \\
57 a\end{array}$ & $\begin{array}{l}0,3 \\
56\end{array}$ & $\begin{array}{l}0,8 \\
65 b\end{array}$ & $\begin{array}{l}- \\
0,8 \\
31 b\end{array}$ & $\begin{array}{l}- \\
0,8 \\
25 b\end{array}$ & $\begin{array}{l}0,5 \\
68 a\end{array}$ & $\begin{array}{l}0,6 \\
73 a\end{array}$ & $\begin{array}{l}0,0 \\
83\end{array}$ & $\begin{array}{l}0,0 \\
53\end{array}$ & $\begin{array}{l}0,0 \\
28\end{array}$ & $\begin{array}{l}0,0 \\
16\end{array}$ & $\begin{array}{l}- \\
0,0 \\
16\end{array}$ & $\begin{array}{l}- \\
0,5 \\
87 a\end{array}$ & $\begin{array}{l}0,0 \\
57\end{array}$ & $\begin{array}{l}- \\
0,5 \\
37 a\end{array}$ & $\begin{array}{l}- \\
0,9 \\
60 b\end{array}$ & $\begin{array}{l}0,9 \\
77 b\end{array}$ & $\begin{array}{l}- \\
0,9 \\
02 b\end{array}$ & 1 & & & & & \\
\hline CRE & $\begin{array}{l}0,9 \\
40 b\end{array}$ & $-0,940 b$ & $\begin{array}{l}- \\
0,6 \\
04 a \\
\end{array}$ & $\begin{array}{l}- \\
0,8 \\
05 b\end{array}$ & $\begin{array}{l}- \\
0,7 \\
06 a\end{array}$ & $\begin{array}{l}- \\
0,2 \\
03\end{array}$ & $\begin{array}{l}0,4 \\
83\end{array}$ & $\begin{array}{l}- \\
0,1 \\
79 \\
\end{array}$ & $\begin{array}{l}- \\
\mathbf{0 , 7} \\
\mathbf{0 9 a}\end{array}$ & $\begin{array}{l}- \\
0,5 \\
87 a\end{array}$ & $\begin{array}{l}- \\
0,9 \\
86 b\end{array}$ & $\begin{array}{l}- \\
0,9 \\
63 b\end{array}$ & $\begin{array}{l}0,2 \\
31\end{array}$ & $\begin{array}{l}0,0 \\
36\end{array}$ & $\begin{array}{l}- \\
0,0 \\
36 \\
\end{array}$ & $\begin{array}{l}0,6 \\
09 a\end{array}$ & $\begin{array}{l}- \\
0,2 \\
03\end{array}$ & $\begin{array}{l}- \\
0,5 \\
61 a\end{array}$ & $\begin{array}{l}0,0 \\
16\end{array}$ & $\begin{array}{l}- \\
0,0 \\
65\end{array}$ & $\begin{array}{l}0,1 \\
41\end{array}$ & $\begin{array}{l}0,08 \\
2\end{array}$ & 1 & & & & \\
\hline CRH & $\begin{array}{l}- \\
0,6 \\
12 a\end{array}$ & $0,612 a$ & $\begin{array}{l}0,9 \\
37 b\end{array}$ & $\begin{array}{l}0,2 \\
27\end{array}$ & $\begin{array}{l}0,7 \\
90 a\end{array}$ & $\begin{array}{l}0,5 \\
02 a\end{array}$ & $\begin{array}{l}- \\
0,1 \\
35\end{array}$ & $\begin{array}{l}- \\
0,5 \\
01 a\end{array}$ & $\begin{array}{l}0,5 \\
62 a\end{array}$ & $\begin{array}{l}0,5 \\
66 a\end{array}$ & $\begin{array}{l}0,3 \\
35\end{array}$ & $\begin{array}{l}0,0 \\
95\end{array}$ & $\begin{array}{l}0,8 \\
44 b\end{array}$ & $\begin{array}{l}\mathbf{0 , 9} \\
33 b\end{array}$ & $\begin{array}{l}- \\
\mathbf{0 , 9} \\
\text { 33b }\end{array}$ & $\begin{array}{l}- \\
0,6 \\
71 a\end{array}$ & $\begin{array}{l}0,9 \\
92 b\end{array}$ & $\begin{array}{l}- \\
0,4 \\
68\end{array}$ & $\begin{array}{l}- \\
0,2 \\
96\end{array}$ & $\begin{array}{l}- \\
0,0 \\
80\end{array}$ & $\begin{array}{l}- \\
0,4 \\
40\end{array}$ & $\begin{array}{l}0,01 \\
8\end{array}$ & $\begin{array}{l}- \\
0,3 \\
25\end{array}$ & 1 & & & \\
\hline TG & $\begin{array}{l}0,9 \\
83 b\end{array}$ & $-\mathbf{- 0 , 9 8 3 b}$ & $\begin{array}{l}- \\
0,9 \\
14 b\end{array}$ & $\begin{array}{l}- \\
0,7 \\
09 a \\
\end{array}$ & $\begin{array}{l}- \\
0,8 \\
88 b \\
\end{array}$ & $\begin{array}{l}- \\
0,3 \\
66 \\
\end{array}$ & $\begin{array}{l}0,3 \\
74\end{array}$ & $\begin{array}{l}0,0 \\
99\end{array}$ & $\begin{array}{l}- \\
0,7 \\
64 a \\
\end{array}$ & $\begin{array}{l}- \\
0,6 \\
76 a \\
\end{array}$ & $\begin{array}{l} \\
0,8 \\
62 \mathrm{~b} \\
\end{array}$ & $\begin{array}{l}- \\
0,7 \\
22 a \\
\end{array}$ & $\begin{array}{l}- \\
0,2 \\
73 \\
\end{array}$ & $\begin{array}{l}- \\
0,4 \\
56 \\
\end{array}$ & $\begin{array}{l}0,4 \\
56\end{array}$ & $\begin{array}{l}0,7 \\
49 a\end{array}$ & $\begin{array}{l}- \\
0,6 \\
52 a\end{array}$ & $\begin{array}{l}- \\
0,1 \\
74 \\
\end{array}$ & $\begin{array}{l}0,1 \\
24\end{array}$ & $\begin{array}{l}0,0 \\
36\end{array}$ & $\begin{array}{l}0,2 \\
89\end{array}$ & $\begin{array}{l}0,08 \\
9\end{array}$ & $\begin{array}{l}0,8 \\
73 b\end{array}$ & $\begin{array}{l}- \\
0,74 \\
4 a\end{array}$ & 1 & & \\
\hline FRAP & $\begin{array}{l}0,7 \\
37 \mathbf{a}\end{array}$ & $-0,737 a$ & $\begin{array}{l}- \\
0,4 \\
22\end{array}$ & $\begin{array}{l}- \\
0,9 \\
90 b\end{array}$ & $\begin{array}{l}- \\
0,2 \\
11\end{array}$ & $\begin{array}{l}0,4 \\
44\end{array}$ & $\begin{array}{l}- \\
0,1 \\
49\end{array}$ & $\begin{array}{l}- \\
0,7 \\
17 a\end{array}$ & $\begin{array}{l}- \\
0,1 \\
21 \\
\end{array}$ & $\begin{array}{l}0,0 \\
39\end{array}$ & $\begin{array}{l} \\
0,6 \\
66 a\end{array}$ & $\begin{array}{l}- \\
0,7 \\
02 a\end{array}$ & $\begin{array}{l}0,3 \\
26\end{array}$ & $\begin{array}{l}0,1 \\
81\end{array}$ & $\begin{array}{l}- \\
0,1 \\
81 \\
\end{array}$ & $\begin{array}{l}0,0 \\
14\end{array}$ & $\begin{array}{l}0,0 \\
30\end{array}$ & $\begin{array}{l}- \\
0,8 \\
34 b\end{array}$ & $\begin{array}{l}- \\
0,6 \\
16 a\end{array}$ & $\begin{array}{l}0,5 \\
30 a\end{array}$ & $\begin{array}{l}- \\
0,5 \\
05\end{array}$ & $\begin{array}{l}0,67 \\
3 a\end{array}$ & $\begin{array}{l}0,7 \\
78 a\end{array}$ & $\begin{array}{l}- \\
0,08 \\
8\end{array}$ & $\begin{array}{l}0,61 \\
9 a\end{array}$ & 1 & \\
\hline $\mathrm{ROH}$ & $\begin{array}{l}- \\
0,7 \\
87 a\end{array}$ & $0,787 a$ & $\begin{array}{l}0,6 \\
40 a\end{array}$ & $\begin{array}{l}0,3 \\
09\end{array}$ & $\begin{array}{l}0,9 \\
46 b\end{array}$ & $\begin{array}{l}0,7 \\
62 a\end{array}$ & $\begin{array}{l}- \\
0,8 \\
\mathbf{3 8 b}\end{array}$ & $\begin{array}{l}- \\
0,4 \\
59\end{array}$ & $\begin{array}{l}0,9 \\
93 b\end{array}$ & $\begin{array}{l}0,9 \\
61 b\end{array}$ & $\begin{array}{l}0,8 \\
70 b\end{array}$ & $\begin{array}{l}0,7 \\
64 a\end{array}$ & $\begin{array}{l}0,0 \\
99\end{array}$ & $\begin{array}{l}0,2 \\
62\end{array}$ & $\begin{array}{l}- \\
0,2 \\
62\end{array}$ & $\begin{array}{l}- \\
0,9 \\
62 b\end{array}$ & $\begin{array}{l}0,4 \\
78\end{array}$ & $\begin{array}{l}- \\
0,0 \\
15\end{array}$ & $\begin{array}{l}- \\
0,6 \\
13 a\end{array}$ & $\begin{array}{l}0,5 \\
44 a\end{array}$ & $\begin{array}{l}- \\
0,7 \\
17 a\end{array}$ & $\begin{array}{l}0,47 \\
8\end{array}$ & $\begin{array}{l}- \\
\mathbf{0 , 7} \\
89 a\end{array}$ & $\begin{array}{l}\mathbf{0 , 5 5} \\
\mathbf{0 a}\end{array}$ & $\begin{array}{l}- \\
\mathbf{0 , 8 1} \\
7 \mathbf{b}\end{array}$ & $\begin{array}{l}- \\
0, \\
23 \\
9\end{array}$ & 1 \\
\hline
\end{tabular}




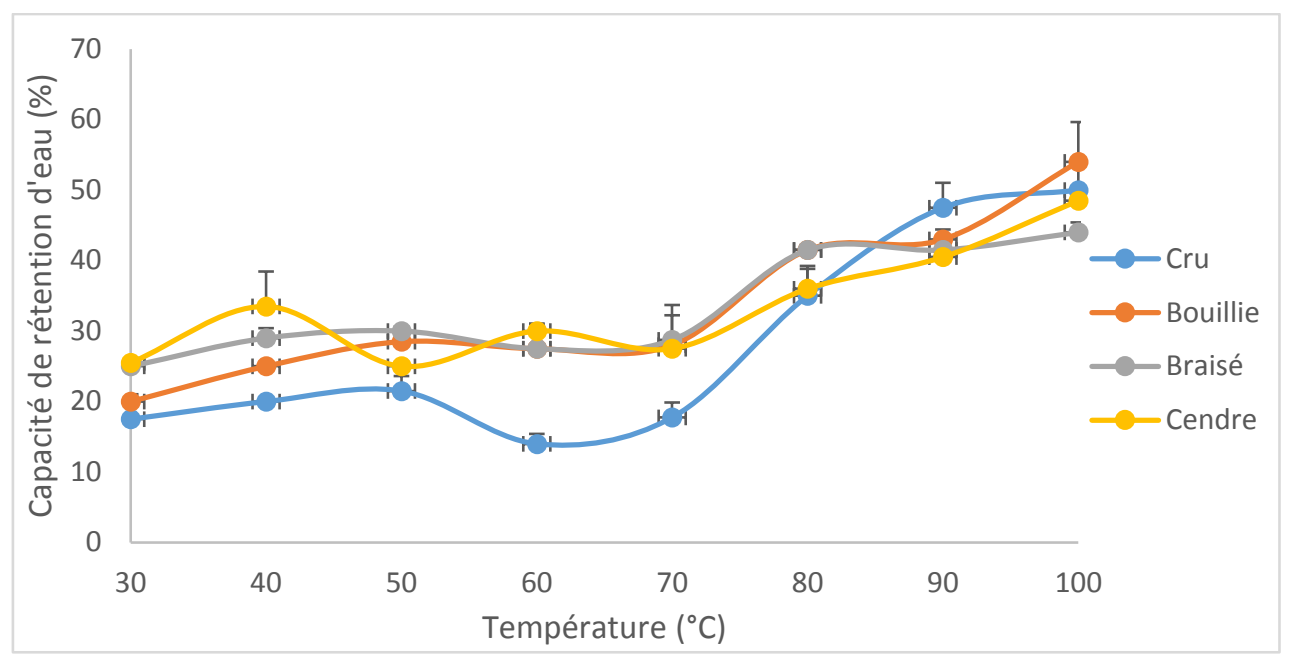

Figure 4 : Capacité de rétention d'eau des différents échantillons en fonction de la température.

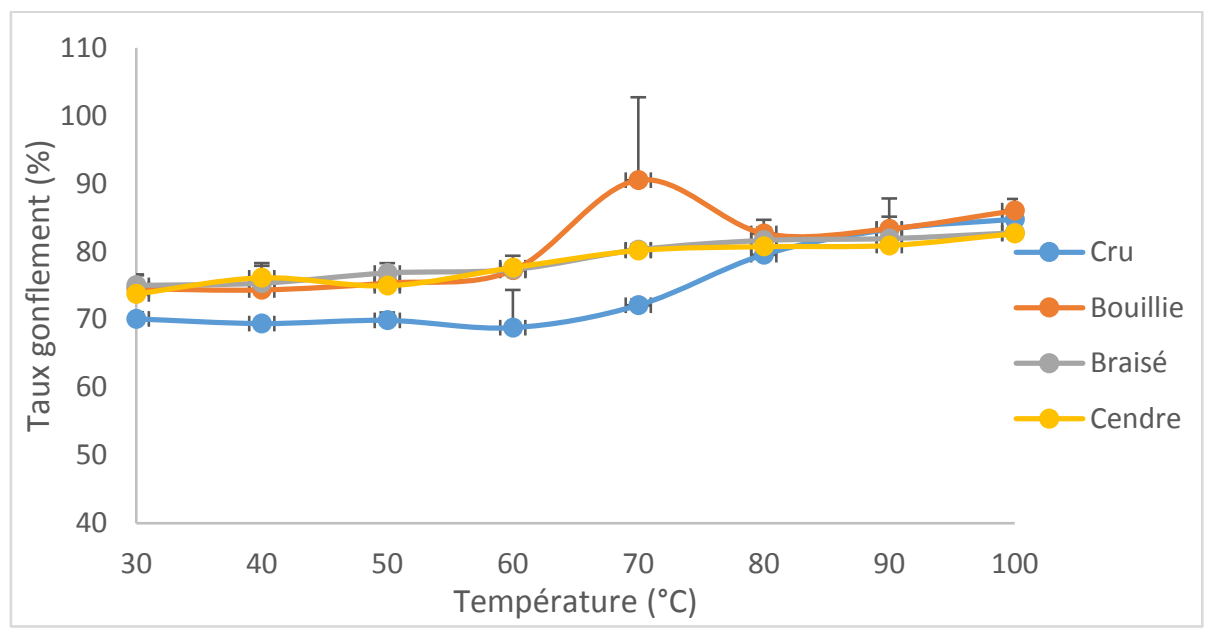

Figure 5 : Taux de gonflement des différents échantillons en fonction de la température.

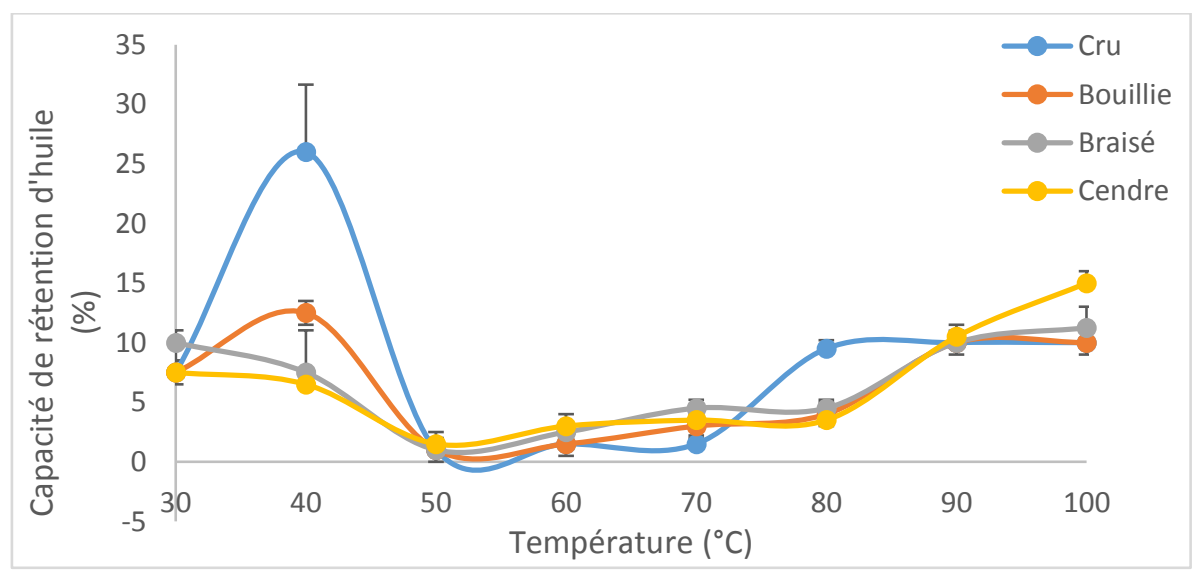

Figure 6 : Capacité de rétention d'huile des différents échantillons en fonction de la température. 


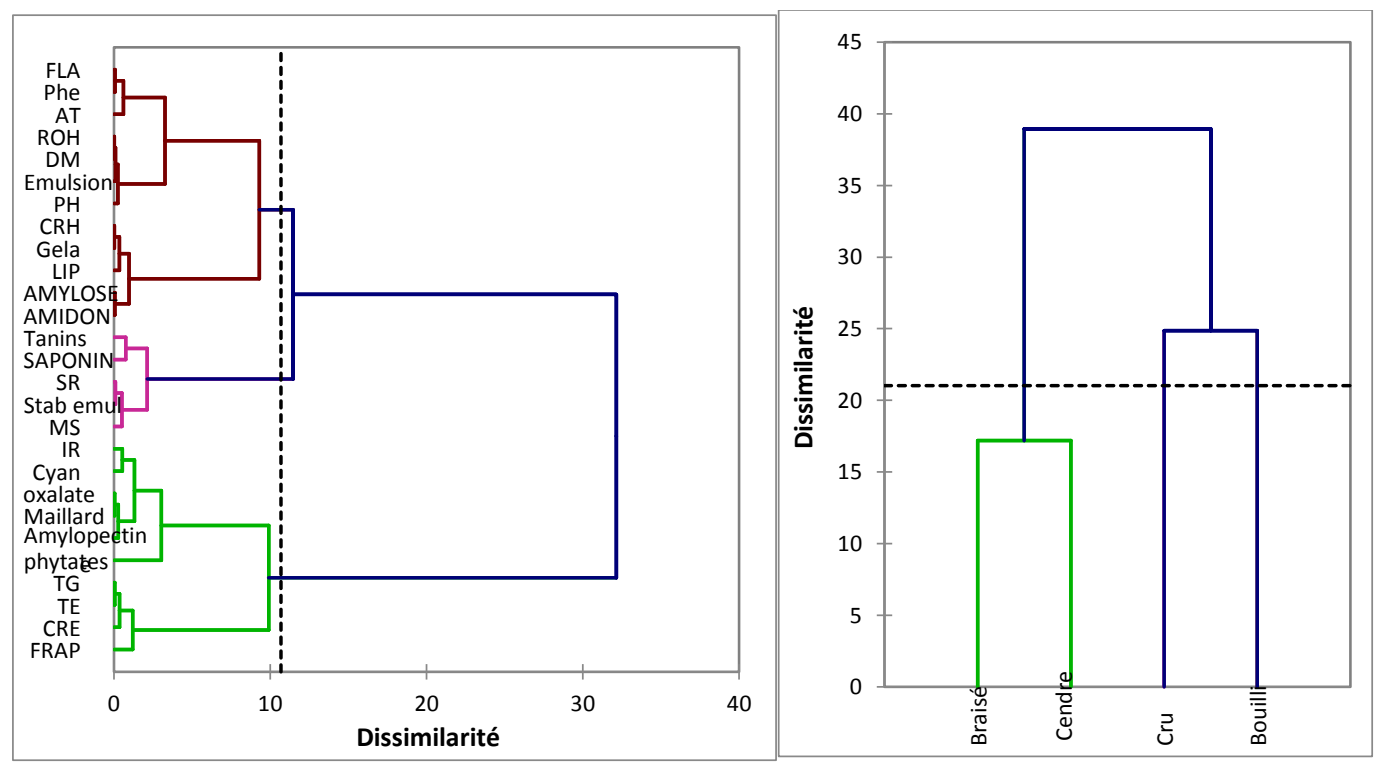

Figure 7 : Dendrogramme des variables (a) et des observations (b).

\section{DISCUSSION}

\section{Effets des différents traitements culinaires sur la composition chimique approximative du "Sapa"}

La teneur en eau des ignames variait de 2,07\% (braisé) à 5,32\% (bouilli). En effet, au cours de la braise on assiste à une élimination de l'eau par évaporation suite à une diffusion par entrainement hors de l'aliment sous l'effet de la chaleur. Par contre, au cours de l'ébullition on assiste à une absorption de l'eau de cuisson ceci par le phénomène de diffusion passive. Les teneurs en eau obtenues ont été toutes inférieures à $14 \%$ et traduirait ainsi une bonne stabilité au stockage. Les valeurs obtenues sont inférieures à ceux de Klang et al. (2019) qui étaient respectivement de $9,95 \%$ et $10,95 \%$ dans les farines de manioc et de pomme de terre. Cela serait dû au temps et à la température de séchage appliqués aux échantillons. La valeur la plus faible a été obtenue avec l'échantillon cuit par ébullition conséquence d'une élimination par lessivage. Klang et al. (2019) ont également observés le même phénomène dans la pomme de terre ayant subi le blanchiment. Pour ce qui est des échantillons braisés et cuits à la cendre, l'élimination de l'eau a entrainé une augmentation de la teneur en lipides ( $\mathrm{r}=-0,837$; $\mathrm{p}<0,01)$. Les valeurs obtenues sont supérieures à celles de Mitiku et Teka (2017) qui étaient de $1,25 \%$ dans la patate variété berkume. Cette différence serait liée à la nature de la plante et aux conditions de culture.

La valeur du cyanure significativement abaissée par la braise $(\mathrm{p}<0,05)$ serait due à une élimination par évaporation et une destruction sous l'effet de la chaleur (Tambo et al., 2019a,b). Les valeurs obtenues sont similaires à celles de Klang et al. (2020), qui étaient de $7,40 \mathrm{mg}$ de $\mathrm{HCN} / \mathrm{kg}$ de MS. Les valeurs obtenues ont été inférieures à la valeur seuil (10 mg d'équivalent $\mathrm{HCN} / \mathrm{Kg}$ de produit au maximum) recommandée par la FAO et l'OMS pour les produits alimentaires.

La réduction de la teneur en amidon et en amylose suite à la braise serait lié à une hydrolyse des liaisons osidiques maintenant la structure de ces molécules avec libération des sucres réducteurs. Ces résultats n'ont pas été similaires à ceux de Tambo et al. (2019b) qui ont démontré que la torréfaction du manioc n'affectait pas significativement la teneur en ces nutriments. Cela serait lié à la température 
et à la durée de la cuisson par la braise. La valeur la plus élevée en sucre réducteur observée avec l'échantillon cuit à la braise et la plus faible avec celle cuit par ébullition serait due à l'élimination de ces sucres solubles dans l'eau de cuisson lors de l'ébullition et d'autre part à l'hydrolyse des liaisons osidiques de l'amylose lors de la braise.

\section{Effets des différents traitements culinaires sur la composition approximative en quelques antinutriments et composés biologiquement actifs du "Sapa"}

La variation de la teneur en saponine observée en fonction des traitements culinaires s'expliquerait par une décomplexation des saponines liées aux nutriments suivit d'une élimination par lessivage dans l'eau de cuisson. Les valeurs obtenues ont été significativement augmentées par les traitements probablement due à une rupture des membranes cellulaires sous l'effet de la chaleur suivi d'une libération de ces composés de la zone insoluble des ignames. En effet, les flavonoïdes et les phénols sont présents dans la plante et lié de manière covalente à certains nutriments à l'instar des protéines. Les teneurs en flavonoïdes obtenues sont de loin inférieures (30 fois) à ceux de Kouame et al. (2021) ayant travaillé sur les jeunes feuilles non encore ouvertes de Piliostigma thonningii preuve que les tubercules et racines ne sont pas des sources prioritaires en composés bioactifs. Les résultats liés aux Phytates et oxalates ont montré que les complexes insolubles formés entre ces anti nutriments et les mineraux dans la plante sont rompues sous l'effet de la chaleur lors des différents traitements suivi d'une élimination des antinutriments (Dongmo et al., 2020). En effet, lors de l'ébullition et de la braise, ceux-ci sont éliminés par lessivage et évaporation respectivement. Les valeurs de phytates obtenues sont supérieures à celles obtenues par Mitiku et Teka (2017) dans la patate. Cela s'expliquerait par la nature du tubercule et les conditions de culture. Il ressort que les valeurs obtenues sont acceptables pour le consommateur (inférieures à $250 \mathrm{mg} .100 \mathrm{~g}$ de MS) et que les deux traitements suscités sont les meilleurs dans la réduction de ces antinutritionnels (Fotso et al., 2020).

Effet des traitements culinaires sur la capacité inhibitrice du radical hydroxyle

La capacité inhibitrice du radical hydroxyle des extraits des différents échantillons qui croit avec la concentration en extrait jusqu'à atteindre son maximum à 200 $\mu \mathrm{g} / \mathrm{ml}$ s'expliquerait par leur teneur élevée en composés phénoliques et en flavonoïdes qui sont libérés lors des traitements thermiques (Dieng et al., 2017 ; Bamba et al., 2021). En effet, le traitement thermique augmente la perméabilité membranaire des tissus (Kettawan et al., 2011). Des études précédentes ont révélé que le pouvoir anti-oxydant des champignons (Lentinula edodes) augmentait après un traitement à $121{ }^{\circ} \mathrm{C}$ pendant $30 \mathrm{~min}$ (Choi et al., 2006) et que la cuisson par la grillade améliorait le plus cette capacité. Ces résultats montrent que la braise préserve au mieux les propriétés inhibitrices du radical hydroxyl et serait par conséquent celle conseillé en vue de la prévention des maladies cardiovasculaires et dérivés issues de l'alimentation.

\section{Effet des traitements culinaires sur le pouvoir réducteur de l'ion fer III}

Le pouvoir réducteur des extraits des échantillons crus, bouillis et braisés qui croit avec la concentration jusqu'à atteindre le pic à la concentration la plus élevée s'expliquerait par une différence de mécanisme d'action. De plus, la capacité la plus faible a été observée avec l'échantillon braisé. Cela serait lié à la présence en quantité importante de phytates $(r=-0,717 ; \mathrm{p}<0,05)$ et de tanins $(r=-0,834$; $\mathrm{p}<0.01)$ dans cet échantillon. En effet, les tanins ont la capacité de complexer certains composés à l'instar des protéines entrainant ainsi la réduction de leur disponibilité et par conséquent de leur pouvoir anti-oxydant. En outre, cela pourrait s'expliquer soit par une élimination des flavonoïdes, saponines et composés phénoliques par évaporation, soit par un changement de conformation de la structure de ces composés suite à ce traitement. 
Roncero-Ramos et al. (2017) ont également rapporté que le pouvoir réducteur de l'ion $\mathrm{Fe}^{3+}$ dans les champignons était abaissé par la grillade.

\section{Indicateur non spécifique de la réaction de Maillard développé lors des traitements culinaires}

L'absorbance la plus importante de l'échantillon cru suivi de l'échantillon cuit à la cendre serait dû à une élimination accrue des sucres réducteurs et acides aminés libres dont la condensation à la surface est responsable de la formation des mélanoïdes lors de l'ébullition. Roncero-Ramos et al. (2017), ont également rapporté le même phénomène dans le champignon cuit par ébullition. Quant-aux échantillons braisé et cuit à la cendre, les valeurs observées seraient liées respectivement à une destruction des acides aminés lors du traitement et une inhibition de la réaction de maillard par les sulfites présents dans la cendre. Les valeurs obtenues sont inférieures à ceux de Roncero-Ramos et al. (2017) dans le champignon cuit au micro-onde.

\section{Caractérisation physique et fonctionnelle des différents échantillons Caractérisation physique}

Les résultats de l'acidité titrable sont dus au fait que, les traitements thermiques fragilisent les parois cellulaires ce qui entrainent la libération des acides organiques et par conséquent l'acidité libre des échantillons. De plus, les acides organiques sont présents dans la plante sous la forme liée à certains nutriments comme les lipides $(\mathrm{r}=0,355)$, amidon $(\mathrm{r}=0,368)$ et une hydrolyse de ces complexes lors des traitements thermiques entrainerait la libération de ces acides organiques et par conséquent de leur augmentation.

La densité massique qui est le rapport entre la masse et le volume occupé par un aliment et qui influence sur le conditionnement et la prise alimentaire (Odadeji et Oyeleke, 2011) est positivement influencé par la taille des particules, la composition nutritionnelle notamment en protéines et lipides (Adebowale et al., 2008; Tambo et al., 2019a). Cela serait lié à la teneur élevée en nutriments notamment lipides et à la taille des particules qui serait augmentée par ces deux traitements. Les valeurs obtenues sont supérieures à celui de Igbokwe et al. (2016) qui était compris entre $0,73-0,81 \mathrm{~g} / \mathrm{ml}$ dans la pomme de terre blanchie. De plus il est à noter que les densités massiques obtenues ont toutes été supérieures à $0,50 \mathrm{~g} / \mathrm{ml}$ qui est celle recommandée pour la préparation des farines infantiles (Klang et al., 2019). Ces résultats montrent donc que cette variété d'igname pourrait être utilisée comme agent épaississant dans la pâtisserie.

L'activité émulsifiante était comprise entre $56,66 \%$ (cru) et 76,66\% (bouillie). Elle est significativement $(\mathrm{p}<0,05)$ améliorée par les traitements culinaires. Cela s'expliquerait par les teneurs élevées en amidon et amylose qui augmenteraient la viscosité empêchant ainsi la coalescence et dont la séparation des phases. Cela serait également lié à la petitesse de la taille des gouttelettes, à la présence des acides organiques qui peuvent modifier la charge à la surface des protéines favorisant ainsi un changement de conformation et donc la capacité émulsifiante (Waidyarathna et Ekanayake, 2021). Les valeurs obtenues étaient supérieures à celles de Harijono et al. (2013) ; Waidyarathna et Ekanayake (2021), qui étaient respectivement de $44,60 \%$ dans l'igname Dioscorea alata et de $40-45 \%$ dans différentes variétés de farines de patate douce. Ce qui s'expliquerait par la charge presque nulle des acides aminés présentes dans cette igname du fait du $\mathrm{pH}$ des farines proches de la neutralité. La stabilité des émulsions variait entre $65 \%$ (bouillie) et $78,45 \%$ (braisé). Elle a été significativement $(\mathrm{p}<0,05)$ augmentée par la braise. La forte stabilité des émulsions observée avec l'échantillon braisé serait liée à sa teneur élevée en sucres réducteurs qui est un composé soluble. Ces observations permettent de relever une possible application de ces échantillons dans la formulation des aliments riches aussi bien en composés lipophile qu'hydrophile. La température de gélatinisation est comprise entre $72-76^{\circ} \mathrm{C}$ et n'a pas été significativement influencé par le 
traitement. Elle est inférieure à celle de Harijono et al. (2013), qui variait entre 80.90 et $91.80{ }^{\circ} \mathrm{C}$. Elle serait certainement liée à la taille des particules, à la composition nutritionnelle notamment en lipides, amylose et protéines. En effet, Baah (2009) a démontré que la présence des lipides à la surface de l'amylose réduisait la vitesse de transfert de chaleur augmentant de ce fait la température de gélatinisation; il en est de même pour la taille des particules. L'indice de reconstitution n'est pas significativement réduit par l'ébullition mais par la braise et la cuisson à la cendre. En effet, l'échantillon cru a présenté l'indice de reconstitution le plus élevée $(73,65 \%)$ contrairement à la braise qui a présenté la valeur la plus faible $(52,27 \%)$. Cela s'expliquerait par la teneur élevée en lipides et en composés phénoliques qui formeraient des complexes insolubles avec l'amidon l'empêchant ainsi de se reconstituer.

Capacité de Rétention d'Eau (CRE)

A des températures élevées, les ponts hydrogènes stabilisant la structure semicristalline des amidons s'ouvrent, se rompent et sont remplacés par des molécules d'eau (Tester et Karkalas, 1996). Ceci justifierait l'augmentation de la quantité d'eau retenue lors de la gélatinisation avec la température.

\section{Taux de gonflement $(\mathrm{Tg})$}

L'augmentation du taux de gonflement avec la température en fonction du traitement culinaire serait liée à une rupture des interactions hydrogènes stabilisant la structure semi-cristalline de l'amidon qui devient amorphe, s'imbibe d'eau et gonfle (Ratnayake et al., 2002). L'échantillon bouilli par contre a présenté un pic de gonflement à $70^{\circ} \mathrm{C}$ suivi d'une chute au-delà de cette température. Cette chute serait la conséquence d'une hydrolyse des molécules d'amylose avec libération des molécules de faible poids moléculaire entrainant de ce fait la baisse du gonflement. En outre, Fetuga et al. (2014), ont démontré que le blanchiment améliorait le taux de gonflement de la farine de patate.

\section{Capacité de rétention d'huile (CRH)}

Il est à noter que l'échantillon cru a présenté la plus forte capacité de rétention d'huile; ce qui s'expliquerait par sa teneur élevée en amidon, lipides et en amylose. En effet, les teneurs en amidon, amylose et phytates présentes dans ces échantillons témoignent du rôle que jouent ces molécules dans la rétention d'huile et des flaveurs des formulations (Adebowale et al., 2005). En effet, les granules d'amidons peuvent fixer grâce aux interactions hydrogènes les molécules lipidiques au niveau de leur portion hydrophile. La faible capacité de rétention d'huile observée avec les traitements serait liée à la perte de certains acides aminés lors du traitement et la formation des mélanoïdines qui sont des inhibiteurs de la rétention d'huile.

\section{Influence des traitements culinaires sur les propriétés physico-chimiques, fonctionnelles et anti-oxydantes : Classification Hiérarchique Ascendante (CAH)}

Dans le premier groupe, une forte reconstitution est caractérisée par une teneur élevée en amylose. L'amylose contrairement à l'amylopectine possède une faible structure cristalline, ce qui la rend fragile face aux procédés hydrothermiques facilitant ainsi son gonflement après rétention d'eau. L'influence négative des teneurs en phytates et oxalates sur ce paramètre serait lié à leur capacité à complexer les cations divalents associés aux ions phosphates présent à la surface de l'amylose et pouvant établir des liaisons ioniques avec les molécules d'eau (Dongmo et al., 2020). Il découle en outre qu'il existe une corrélation positive mais non significative entre la FRAP et la réaction de maillard ( $\mathrm{r}=$ 0,124). Roncero-Ramos et al. (2017) ont démontré que les produits de la réaction de maillard ont la capacité de réduire le $\mathrm{Fe}^{3+}$. De même, la FRAP a été négativement corrélé aux oxalates $(r=-0,505)$ et aux phytates $(r=-0,707)$. En effet, Mitiku et Teka (2017), ont rapporté que les ces antinutriments sont capables de fixer outre les ions certains composés phénoliques dotés de propriétés anti-oxydantes par des liaisons de coordinance réduisant de ce fait leur disponibilité et leur activité. La corrélation positive $(r=0,483)$ observée entre la teneur en cyanure et la CRE découle du fait 
que les composés cyanogétiques sont présents dans la plante sous forme liée aux glucides. Ces glucides du fait de la présence des groupements -OH à leur surface pourraient donc établir des interactions hydrogènes avec les molécules d'eau et par conséquent leur retenir (Tambo et al., 2019b).

Le deuxième regroupement est constitué de la matière sèche, SR, tanins, saponines et de la stabilité des émulsions. La matière sèche augmente la teneur en saponines $(\mathrm{r}=0,807)$, sucres réducteurs $(\mathrm{r}=0,816)$ et tanins $(r=0,352)$. La stabilité des émulsions est positivement corrélée aux tanins $(\mathrm{r}=0,468)$, saponines $(r=0,697)$ et SR $(r=0,969)$. Ces observations sont contraires à celles de Harijono et al. (2013); Mitiku et Teka (2017), qui ont démontré que les tanins tout comme les saponines sont des antinutriments pouvant complexer et réduire la biodisponibilité des protéines qui interviennent positivement dans la stabilisation des émulsions. Outre cela, les sucres réducteurs étant de faibles poids moléculaires possèdent une hydrophilité réduite et peuvent par conséquent former des micro-couches stabilisatrices entre les interfaces huile/eau. En outre, les tanins et les saponines forment des complexes insolubles dans la plante ce qui expliquerait leur évolution positive la diminution de la teneur en eau. La corrélation positive $(r=0,749)$ observée entre les saponines et les tanins montre que la synthèse de ces deux éléments par la plante serait sous la direction d'un même gène.

Dans le troisième groupe, les composés phénoliques en général (flavonoïdes et autres) possèdent à leur surface des groupements $-\mathrm{OH}$ qui ont des hydrogènes très électrophiles pouvant facilement se détacher (ou être capter par les radicaux libres) de la molécule d'oxygène pour se fixer et stabiliser le radical hydroxyle (Roncero-Ramos et al., 2017). La densité massique évolue positivement avec les lipides $(r=0,612)$, les phénols $(r=0,616)$ et la CRH $(r=0,562)$. La densité massique est positivement influencée par la taille des particules et la composition nutritionnelle (Adebowale et al., 2008). En effet, l'abondance des molécules de haut poids moléculaire comme les protéines et les composés phénoliques dans l'aliment augmente la densité massique. La CRH est une propriété fonctionnelle améliorée par la présence des acides aminés hydrophobes au niveau de la structure des protéines. Hors ces dernières évoluent positivement avec la densité massique d'où la corrélation positive avec la CRH. La CRH quant à elle, est corrélée au pH ( $\mathrm{r}=0,790)$, l'amidon $(\mathrm{r}=0,844)$, l'amylose $(\mathrm{r}=0,933)$ et l'acidité titrable $(\mathrm{r}=0,502)$. Le $\mathrm{pH}$ et les acides organiques modifient la tension des surfaces des molécules contribuant ainsi à réduire les forces de répulsion entre les molécules de nature opposée au niveau de leurs surfaces respectives. C'est le cas de l'amidon et de l'amylose. La corrélation positive observée entre le $\mathrm{pH}$ et les phénols $(\mathrm{r}=0,367)$ a également été rapportée par Guedes et al. (2011), dans les peaux de pomme de terre. La température de gélatinisation est positivement corrélée à la densité massique $(r=0,504)$, l'amidon $(\mathrm{r}=0,904)$ et à l'amylose $(\mathrm{r}=0,969)$. Harijono et al. (2013) ont également rapporté que la température de gélatinisation croissait proportionnellement avec la taille des granules, les teneurs en protéines, amidon et amylose.

\section{Conclusion}

La contribution à la valorisation de $D$. praehensilis au Cameroun à travers la caractérisation des différents traitements culinaires en fonction de quelques propriétés physicochimiques et antinutritionnelles a montré que les teneurs en amidon et en amylose étaient significativement élevées par ébuillition et par cuisson à la cendre contrairement à l'amylopectine. La valeur la plus élevée en sucre réducteur est l'échantillon cuit à la braise. La teneur en saponines a été significativement abaissée par l'ébullition et les teneurs en composes phénoliques et en flavonoïdes ont été significativement augmentées par les traitements bien que, l'échantillon cru a présenté la teneur en phytates la plus élevée. L'augmentation de la concentration des extraits entraine une augmentation de la capacité inhibitrice du radical hydroxyle des extraits des différents 
échantillons. Tous les paramètres étudiés ont permis de distinguer trois groupes et permettent de conclure que $D$. praehensilis a une diversité physicochimique et antioxydante très élevée rendant possible tout programme visant la sélection et l'amélioration variétale au sein de cette espèce, ce qui contribue ainsi à promouvoir sa domestication, sa vulgarisation et la conservation de sa biodiversité au Cameroun.

\section{CONFLIT D'INTERETS}

Les auteurs déclarent qu'ils n'ont pas d'intérêts concurrents.

\section{CONTRIBUTIONS DES AUTEURS}

SRPK a collecté les échantillons de SAPA, analysé en laboratoire et contribué à la rédaction. Il était accompagné de STT au laboratoire dans la rédaction. MM a orienté les travaux et contribué à la rédaction. JRN et YPM ont relu et conseillé scientifiquement.

\section{REMERCIEMENTS}

Les auteurs remercient la communauté du village Payo, l'URBPMAN de l'université de Dschang, le mécanisme Indigenous Peoples Assistance Facility (IPAF) et Samburu Women Trust (SWT) et le Programme des Nations Unies pour le Développement (PNUD).

\section{REFERENCES}

Adebowale A, Sanni S, Oladapo F. 2008. Chemical, functional and sensory properties of instant yam breadfruit flour. Nig. Food J., 26(1): 2-12.

Adebowale YA, Adeyemi IA, Oshodi AA. 2005. Functional and physicochemical properties of flour of six Mucuna species. Afr. J. Biot., 4(12): 1461-1468.

Bahorun T, Soobrattee MA, Luximon-Ramma V, Aruoma OI. 2006. Free radicals and antioxidants in cardiovascular health and disease. Int. J. Med. Upd., 1(2): 25-41.

Bamba B, Benie CKD, Ouattara A, Doukourou DN, Kamou KR, Ouattara K. 2021. Teneurs en phénols totaux, activités antioxydantes des macérés et décocté des feuilles de Uvaria chamae P. Beauv.
(Annonaceae). Int. J. Biol. Chem. Sci., 15(1): 54-67. DOI: https://dx.doi.org/10.4314/ijbcs.v15i1.6

Beuchat LR. 1977. Functional and electrophoretic characteristics of succinylated peanut flour protein. J. Agr. Food chem., 25(2): 258-261.

Cabrera-Millet M, López-Martínez N, Michaux S. 1984. Un exemple de lignée endémique ibéroccitane, les campagnols Microtus brecciensis et Microtus cabrerae (Mammalia, Rodentia): étude phyogénétique et contexte écologique d'un phénomène évolutif récent. In Symposium paléontologique Georges Cuvier; 69-83.

Choi Y, Lee SM, Chun J, Lee HB, Lee J. 2006. Influence of heat treatment on the antioxidant activities and polyphenolic compounds of Shiitake (Lentinus edodes) mushroom. Food chem., 99(2): 381-387.

Dieng SIM, Fall AD, Diatta-Badji K, Sarr A, Sene M, Sene M, Mbaye A, Diatta W, Bassene E. 2017. Evaluation de l'activité antioxydante des extraits hydroethanoliques des feuilles et écorces de Piliostigma thonningii Schumach. Int. J. Biol. Chem. Sci., 11(2): 768-776. DOI: https://dx.doi.org/10.4314/ijbcs.v11i2.19

Dongmo H, Tambo TS, Teboukeu BG, Mboukap NA, Fotso SB, Djuidje TMC, Klang JM. 2020. Effect of process and variety on physico-chemical and rheological properties of two new corn flour varieties (Atp and Kassaï). J. Agr. Food Res., 2: 10075. DOI: https://doi.org/10.1016/j.jafr.2020.10007 5

Dounias E. 1996. Sauvage ou cultivé? La paraculture des ignames sauvages par les pygmées Baka du Cameroun. IRD, Cameroun. http://horizon.documentation.ird.fr

Doussoh AM, Dangou JS, Houedjissin SS, Assogba AK, Ahanhanzo C. 2016. Analyse des connaissances endogènes et des déterminants de la production de la patate douce [Ipomoea batatas (L.)], une culture à haute valeur socioculturelle et 
économique au Bénin. Int. J. Biol. Chem. Sci., 10(6): 2596-2616. DOI: http://dx.doi.org/10.4314/ijbcs.v10i6.16

Fétéké F, Philippart J. 2008. Plan D'aménagement des Unités Forestières D'aménagement n 10030 et 10031 Regroupées. Cellule d'aménagement Pallisco and Nature Plus, Mindourou, Cameroon and Gembloux, Belgium.

Fetuga GO, Tomlins K, Henshaw FO, Idowu MA. 2014. Effect of variety and processing method on functional properties of traditional sweet potato flour ("elubo") and sensory acceptability of cooked paste ("amala"). Food Sci. Nutr., 2(6): 682-691.

Fotso SB, Tambo TS, Teboukeu BG, Klang JM. 2020. Optimization of the Reduction of Phytates and Trypsin Inhibitors of Soybeans (Glycine Max L.): Effect of Soaking And Cooking. J. Food Nutr. Sci., 7(1): 70-76. DOI: $10.15436 / 2377-$ 0619.20 .2900

Gao X, Ohlander M, Jeppsson N, Björk L, Trajkovski V. 2000. Changes in antioxidant effects and their relationship to phytonutrients in fruits of sea buckthorn (Hippophae rhamnoides L.) during maturation. J. Agr. Food Chem., 48(5): 1485-1490.

Guedes AC, Amaro HM, Pierriera RD, Malcata FX. 2011. Effect of temperature and $\mathrm{pH}$ on growth and antioxidant content of the microalgia scenedesmus obliquus. Biot. Prog., 27(5): 1218-1224.

Harijono TE, Saputri DS, Kusnadi J. 2013. Effect of blanching on properties of water yam (Dioscorea alata) flour. Adv. J. Food Sci. Tech., 5(10): 1342-50.

Igbokwe CJ, Akubor PI, Mbaeyi-Nwaoha IE. 2016. Effect of processing on the chemical composition, phytochemical contents and functional properties of yellow fleshed aerial yam (Dioscorea bulbifera) flour. Inn. J. Food Sci., 4(4): 14.

Jarvis CE, Walker JR. 1993. Simultaneous, rapid, spectrophotometric determination of total starch, amylose and amylopectin. J. Sci. Food Agr., 63(1): 53-57.

Jiménez-Zamora A, Delgado-Andrade C, Rufiàn-Henares JA. 2016. Antioxidant capacity, total phenols and color profile during the storage of selected plants used for infusion. Food Chem., 199: 339-346.

Kettawan A, Chanlekha K, Kongkachuichai R, Charoensiri R. 2011. Effects of cooking on antioxidant activities and polyphenol content of edible mushrooms commonly consumed in Thailand. Pak. J. Nutr., 10: 1094-1103.

Klang JM, Tambo TS, Nguemguo KLG, Teboukeu BG, Ndomou HSC, Kohole FHA, Womeni HM. 2019. Effect of bleaching and variety on the physicochemical, functional and rheological properties of three new Irish potatoes (Cipira, Pamela and Dosa) flours grown in the locality of Dschang (West region of Cameroon). Heliyon, 5(12): e02982. DOI: https://doi.org/10.1016/j.heliyon.2019.e0 2982.

Klang JM, Tambo TS, Matueno KFE, Teboukeu BG, Womeni HM. 2020. Optimization using response surface methodology (RSM) of the energy density of flour-based gruels of sweet cassava (Manihot esculenta Crantz) flour: Effect of the addition of two new sprouted rice varieties produced under optimal conditions (Nerica 3 and Nerica L56). NFS J., 19: 16-25. DOI: https://doi.org/10.1016/j.nfs.2020.04.001

Koziol MJ. 1991. Afrosimetric estimation of threshold saponin concentration for bitterness in quinoa (Chenopodium quinoa Willd). J. Sci. Food Agr., 54(2): 211-219.

Kouamé TK, Siaka S, Kassi ABB, Soro Y. 2021. Détermination des teneurs en polyphénols totaux, flavonoïdes totaux et tanins de jeunes feuilles non encore ouvertes de Piliostigma thonningii (Caesalpiniaceae). Int. J. Biol. Chem. Sci., 15(1): 97-105. DOI: https://dx.doi.org/10.4314/ijbcs.v15i1.9 
Leach HW, McCowen DL, Schoch TJ. 1959. Swelling and solubility patterns of various starches and structure of starch granule. Cer. Chem., 36: 534-544.

Marfak A. 2003. Radiolyse gamma des flavonoïdes, Etude de leur réactivité avec les radicaux issus des alcools: formation de depsides. Mémoire, Université de Limoges, Limoges.

Mbougueng PD. 2009. Influence des amidons natifs ou acétylés de manioc et de pomme de terre sur les propriétés physicochimiques et texturales du pâté de Boeuf (Bos indicus). Doctoral dissertation, Université de Lorraine, Lorraine. https://hal.univ-lorraine.f.

Mitiku DH, Teka TA. 2017. Nutrient and antinutrient composition of improved sweet potato [Ipomea batatas (L) Lam] varieties grown in eastern Ethiopia. Nutr. Food Sci., 47(3): 369-380.

Okaka JC, Okorie PA, Ozo ON. 1991. Quality evaluation of sun-dried yam chips. Trop. Sci., 31(3): 265-275.

Okezie BO, Bello AB. 1988. Physicochemical and functional properties of winged bean flour and isolate compared with soy isolate. J. Food Sci., 53(2), 450-454.

Oyaizu M. 1986. Studies on products of browning reaction. Jap. J. Nutr. Diet., 44(6): 307-315.

Pearce KN, Kinsella JE. 1978. Emulsifying properties of proteins: evaluation of a turbidimetric technique. J. Agr. Food Chem., 26(3): 716-723.

Ratnayake WS, Hoover R, Tom W. 2002. Pea starch: Composistion, structure and properties. Review. Starch/Starke, 54: 217-234.

Reddy NR, Pierson MD, Sathe SK, Salunkhe DK. 1989. Phytates in Cereals and Legumes. CRC Press: États-Unis.

Roncero-Ramos I, Mendiola-Lanao M, PérezClavijo M, Delgado-Andrade C. 2017. Effect of different cooking methods on nutritional value and antioxidant activity of cultivated mushroom. Int. J. Food Sci. Nutr., $\quad$ 68(3): 287-297. $\quad$ DOI: 10.1080/09637486.2016.1244662

Sanginga N, Mbabu A. 2015. Racines et tubercules (Manioc, Igname, pomme de terre et patate douce). Centre International de Recherche Abdou Diouf, Dakar, Sénégal, 1p.

Shekhar S, Mishra D, Buragohain AK, Chakraborty S, Chakraborty N. 2015. Comparative analysis of phytochemicals and nutrient availability in two contrasting cultivars of sweet potato (Ipomoea batatas L.). Food Chem., 173: 957-965.

DOI: https://doi.org/10.1016/j.foodchem.2014. 09.172

Tambo TS, Klang JM, Ndomou HSC, Teboukeu BG, Womeni HM. 2019a. Characterisation of corn, cassava and commercial flours: use of amylases rich flours of germinated corn and sweet potato in the reduction of the consistency of the gruels made from these floursinfluence on the nutritional and energy value. Food Sci. Nutr., 7(4): 1190-1206. DOI: https://doi.org/10.1002/fsn3.902

Tambo TS, Klang JM, Ndomou HSC, Kohole FHA, Womeni HM. 2019b. Application of amylase rich flours of corn and sweet potato to the reduction of consistency of cassava and corn gruels. J. Food Pro. Pres., 43(9): e14058. DOI: https://doi.org/10.1111/jfpp.14058

Tester R, Karkalas J. 1996. Swelling and gelatinization of oat starches. Cer. Chem., 73: $271-273$.

Waidyarathna GRNN, Ekanayake S. 2021. Nutrient composition and functional properties: suitability of flour of sweet potatoes (Ipomea batatas) for incorporation into food production. Int. $J$. Biol. Chem. Sci., 15(3): 897-908. DOI: https://dx.doi.org/10.4314/ijbcs.v15i3.5 